\title{
THE ORIGIN OF HVS17, AN UNBOUND MAIN SEQUENCE B STAR AT 50 kpc
}

\author{
Warren R. Brown ${ }^{1}$, Judith G. Cohen ${ }^{2}$, Margaret J. Geller ${ }^{1}$, and Scott J. Kenyon ${ }^{1}$ \\ ${ }^{1}$ Smithsonian Astrophysical Observatory, 60 Garden Street, Cambridge, MA 02138, USA; wbrown@ cfa.harvard.edu, \\ mgeller@cfa.harvard.edu, skenyon@cfa.harvard.edu \\ ${ }^{2}$ Palomar Observatory, Mail Stop 249-17, California Institute of Technology, Pasadena, CA 91125, USA; jlc@astro.caltech.edu \\ Received 2013 June 3; accepted 2013 July 27; published 2013 August 30
}

\begin{abstract}
We analyze Keck Echellette Spectrograph and Imager spectroscopy of HVS17, a B-type star traveling with a Galactic rest frame radial velocity of $+445 \mathrm{~km} \mathrm{~s}^{-1}$ in the outer halo of the Milky Way. HVS17 has the projected rotation of a main sequence B star and is chemically peculiar, with solar iron abundance and sub-solar alpha abundance. Comparing measured $T_{\text {eff }}$ and $\log g$ with stellar evolution tracks implies that HVS17 is a $3.91 \pm 0.09$ $M_{\odot}, 153 \pm 9$ Myr old star at a Galactocentric distance of $r=48.5 \pm 4.6 \mathrm{kpc}$. The time between its formation and ejection significantly exceeds $10 \mathrm{Myr}$ and thus is difficult to reconcile with any Galactic disk runaway scenario involving massive stars. The observations are consistent, on the other hand, with a hypervelocity star ejection from the Galactic center. We show that Gaia proper motion measurements will easily discriminate between a disk and Galactic center origin, thus allowing us to use HVS17 as a test particle to probe the shape of the Milky Way's dark matter halo.
\end{abstract}

Key words: Galaxy: center - Galaxy: halo - Galaxy: kinematics and dynamics - stars: early-type - stars: individual (SDSS J164156.39+472346.1)

Online-only material: color figures

\section{INTRODUCTION}

The origin of unbound stars in the Milky Way is linked to our understanding of physical processes in the Galaxy. Unbound neutron stars, for example, are likely remnants of asymmetric core-collapse supernova explosions (e.g., Arzoumanian et al. 2002). Unbound white dwarfs, such as US 708 (Hirsch et al. 2005) and LP-400 (Kilic et al. 2013), are likely former binary companions of objects that exploded in double-detonation supernovae (Justham et al. 2009; Wang \& Han 2009; Geier et al. 2013). Unbound main sequence stars, stars that have not exploded, are a new probe of these issues.

There are two models for explaining unbound main sequence stars: runaway ejections from the Galactic disk, and hypervelocity star (HVS) ejections from the Galactic center. Traditional runaway B stars (e.g., Humason \& Zwicky 1947) are either the former binary companions of systems disrupted by supernovae (Blaauw 1961) or ejections in dynamical encounters with other stars (Poveda et al. 1967). Heber et al. (2008) first showed that an unbound runaway can result from an extreme ejection from the outer disk in the direction of disk rotation. The physical size of main sequence stars places a natural speed limit on runaway ejections, however, and so unbound runaways should be rare compared to HVSs (see Bromley et al. 2009).

Hills (1988) predicted that unbound "HVSs" are a natural consequence of a massive black hole (MBH). There is overwhelming evidence for a $4 \times 10^{6} M_{\odot} \mathrm{MBH}$ in the center of the Milky Way (Ghez et al. 2008; Gillessen et al. 2009). Theorists predict that three-body interactions with this MBH will eject unbound HVSs at a rate of $\sim 10^{-4} \mathrm{yr}^{-1}$ (Hills 1988; Yu \& Tremaine 2003; Perets et al. 2007; Zhang et al. 2010). The "S-stars" presently observed in short-period, eccentric orbits around the MBH match expectations for being the former companions of ejected HVSs (Alexander \& Livio 2004; Ginsburg \& Loeb 2006; Perets 2009; Zhang et al. 2013; Madigan et al. 2013).

The first example of an unbound HVS is a short-lived B-type star traveling at twice the Galactic escape velocity at a distance of $\simeq 100 \mathrm{kpc}$ (Brown et al. 2005). After two other serendipitous HVS discoveries (Hirsch et al. 2005; Edelmann et al. 2005), a targeted HVS survey by Brown et al. (2006a, 2006b, 2007a, 2007b, 2009, 2012b) discovered 16 unbound B-type stars and a similar number of possibly bound HVSs. The existing observations - the unbound velocities, the observed number of HVSs, and the Galactic latitude distribution of HVSs-support the MBH ejection picture. A handful of HVSs bright enough for echelle spectroscopy are confirmed main sequence B stars at 50-100 kpc distances (Przybilla et al. 2008b, 2008c; López-Morales \& Bonanos 2008; Brown et al. 2012a). These distances imply $\simeq 100$ Myr elapsed between the HVSs' formation and ejection, a timescale that is difficult to reconcile with any runaway scenario involving supernovae or encounters with massive stars that live for $\simeq 10 \mathrm{Myr}$, but consistent with the expected timescale for dynamical interactions of stars with the central MBH (Brown et al. 2012a). What remains unclear, however, is whether all of the claimed HVSs are Galactic center ejections.

A Galactic center origin for the HVSs links them with tidal disruption events (Bromley et al. 2012). HVSs and tidal disruption events both involve stars formed in the central regions that are scattered into a MBH's "loss cone." Observations imply a tidal disruption rate of $10^{-5} \mathrm{yr}^{-1}$ per galaxy (e.g., van Velzen et al. 2011), however, models are required to interpret the tidal disruption light curves. If HVSs are ejected from the Galactic center, then HVSs provide a direct measure of the stellar interaction rate for a $4 \times 10^{6} M_{\odot} \mathrm{MBH}$.

A Galactic center origin for the HVSs also makes them unique and important probes of the Milky Way's dark matter distribution (Gnedin et al. 2005; Yu \& Madau 2007; Wu et al. 2008). If HVSs are launched from $r=0$, then they integrate the Milky Way's gravitational potential as they travel out to $100 \mathrm{kpc}$ distances. Any deviation of the HVSs' trajectories from the Galactic center thus measures the Milky Way's mass distribution (Gnedin et al. 2005). This measurement requires both proper motions (Brown et al. 2010) and accurate distances. 
Proper motions and distances can also discriminate between an HVS origin from the Galactic center and a runaway origin from the outer disk (Heber et al. 2008; Tillich et al. 2009; Irrgang et al. 2010; Tillich et al. 2011).

High resolution spectroscopy allows us to measure accurate stellar parameters and thus an improved distance. The discriminatory power of these observations is important because evolved blue horizontal branch (BHB) stars can have the same temperature and gravity as late B-type main sequence stars, but with very different luminosities. For example, a BHB star with the temperature and gravity of HVS17 is eight times less luminous (Dorman et al. 1993; Dotter et al. 2007), and thus is located at a 2.8 times nearer distance, than a main sequence B star of the same temperature and gravity. This distinction matters to our flight time and proper motion calculations, as well as to our understanding of the mass function of stars encountering the $\mathrm{MBH}$. Fortunately, projected stellar rotation $v \sin i$ provides a clean discriminant between evolved and main sequence late B-type stars. Evolved BHB stars have median $v \sin i=9 \mathrm{~km} \mathrm{~s}^{-1}$; the most extreme BHB rotation known is $40 \mathrm{~km} \mathrm{~s}^{-1}$ (Behr 2003a). Late B-type main sequence stars, on the other hand, have median $v \sin i=150 \mathrm{~km} \mathrm{~s}^{-1}$; the most extreme main sequence star rotation exceed $350 \mathrm{~km} \mathrm{~s}^{-1}$ (Abt et al. 2002; Huang \& Gies 2006).

Here, we present a study of SDSS J164156.39+472346.1, henceforth HVS17, a newly discovered HVS (Brown et al. 2012 b) bright enough for high resolution spectroscopy with the $10 \mathrm{~m}$ Keck telescope. HVS17 appears to be a chemically peculiar B star, which means that diffusion processes have erased any constraint on stellar origin provided by abundance. Stellar rotation suffers from no such ambiguity, and on this basis we conclude that HVS17 is a main sequence B star at a distance of $50 \mathrm{kpc}$. We investigate HVS17's origin on the basis of trajectory and flight time calculations. If HVS17 is a runaway ejected from the Galactic disk, it requires a minimum ejection of $+415 \mathrm{~km} \mathrm{~s}^{-1}$ to reach its present location and velocity. A Galactic center origin, on the other hand, implies a proper motion that differs from the disk origin by $\simeq 1$ mas $\mathrm{yr}^{-1}$, a difference easily measurable in the near future with Gaia.

In Section 2 we describe the observations and stellar atmosphere analysis. In Section 3 we discuss the nature and origin of HVS17. We conclude in Section 4.

\section{DATA}

\subsection{Observations}

We observed HVS17 using the Echellette Spectrograph and Imager (ESI) spectrograph (Sheinis et al. 2002) at the $10 \mathrm{~m}$ Keck 2 telescope. We used the 0.5 arcsec slit to obtain a spectral resolution of $R \simeq 9000$; the spectral coverage is 3900-9300 $\AA$. We collected seven 30 minute exposures over the course of three nights 2012 April 26-28.

We use the pipeline package MAKEE ${ }^{3}$ to extract a onedimensional spectrum for each echelle order and calibrate the wavelength scale using arc spectra with $\mathrm{Cu}, \mathrm{Xe}, \mathrm{Ne}, \mathrm{Ar}$, and $\mathrm{Hg}$ lines. Comparison of arc and sky lines show that the wavelength shift between the three nights is less than 0.6 pixel $\left(7 \mathrm{~km} \mathrm{~s}^{-1}\right)$. Each exposure of HVS17 was individually processed, and the results summed. Our total integration time of $3.5 \mathrm{hr}$ achieves a

\footnotetext{
3 MAKEE is a spectroscopic reduction package developed by T.A. Barlow. It is freely available from the Keck HIRES home page www2.keck.hawaii.edu/inst/hires.
}

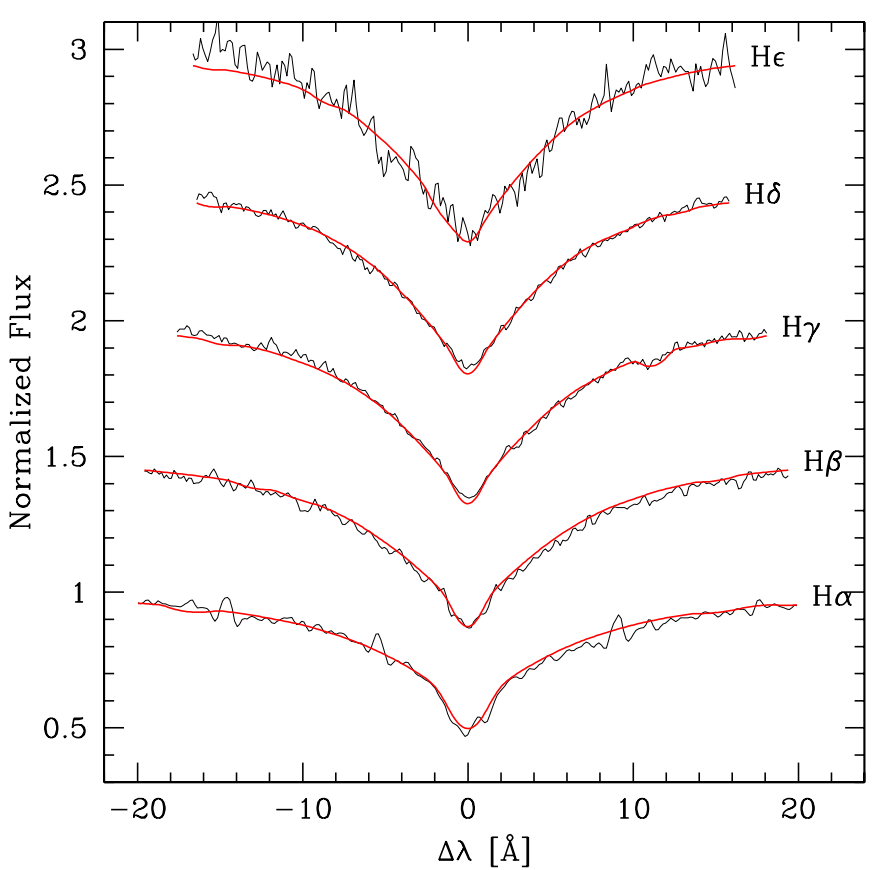

Figure 1. Observed hydrogen Balmer lines compared to the best-fit model (smooth lines). The temperature- and surface gravity-sensitive lines give best-fit values of $T_{\text {eff }}=12350 \pm 290 \mathrm{~K}$ and $\log g=3.80 \pm 0.086$.

(A color version of this figure is available in the online journal.)

signal-to-noise ratio $(\mathrm{S} / \mathrm{N})$ of 300 per spectral resolution element in the continuum at $4500 \AA$.

We also observed four B stars selected from Abt et al. (2002) that span a wide range in projected rotational velocity $\left(15<v \sin i<240 \mathrm{~km} \mathrm{~s}^{-1}\right)$. The stars are HR5833, HR5834, HR6502, and HR6851. We used the high S/N spectra of these four B stars to validate our stellar atmosphere analysis below.

\subsection{Spectral Analysis}

Our spectral analysis methodology is described in Brown et al. (2012a). In a sentence, we calculate synthetic spectra using the SPECTRUM package (Gray \& Corbally 1994) and ATLAS9 ODFNEW model atmosphere grids (Castelli \& Kurucz 2004; Castelli et al. 1997), normalize the continuum, calculate the $\chi^{2}$ of each synthetic model against the data, and then fit the resulting distribution of $\chi^{2}$ to derive the best-fitting parameters and uncertainties.

The best-fit $+248.0 \pm 2.2 \mathrm{~km} \mathrm{~s}^{-1}$ radial velocity is in good agreement with the $+246 \pm 9 \mathrm{~km} \mathrm{~s}^{-1}$ radial velocity measured from medium-resolution spectroscopy at the MMT (Brown et al. $2012 b$ ); there is no evidence for velocity variability. HVS17's heliocentric velocity corresponds to a minimum velocity of $+445 \mathrm{~km} \mathrm{~s}^{-1}$ in the Galactic rest frame (see Brown et al. 2012b).

Next, we measure projected rotation using Mg II $\lambda 4481 \AA$, the single strongest metal line in the spectrum. The minimum $\chi^{2}$ is sensitive to $\mathrm{Mg}$ abundance, but insensitive to $T_{\text {eff }}$ or $\log g$. Iterating with the best-fit parameters below, we find $v \sin i=$ $68.7 \pm 5.4 \mathrm{~km} \mathrm{~s}^{-1}$.

Given the observed $v \sin i$, we measure effective temperature and surface gravity from the $T_{\text {eff }}$ - and $\log g$-sensitive hydrogen Balmer lines. The best-fit values are $T_{\text {eff }}=12,350 \pm 290 \mathrm{~K}$ and $\log g=3.80 \pm 0.086$. Figure 1 compares the best-fit model with the data.

Finally, we measure metal abundances by generating a list of all metal lines with $>10 \mathrm{~m} \AA$ equivalent widths, and then averaging the abundances measured for all lines of a given 

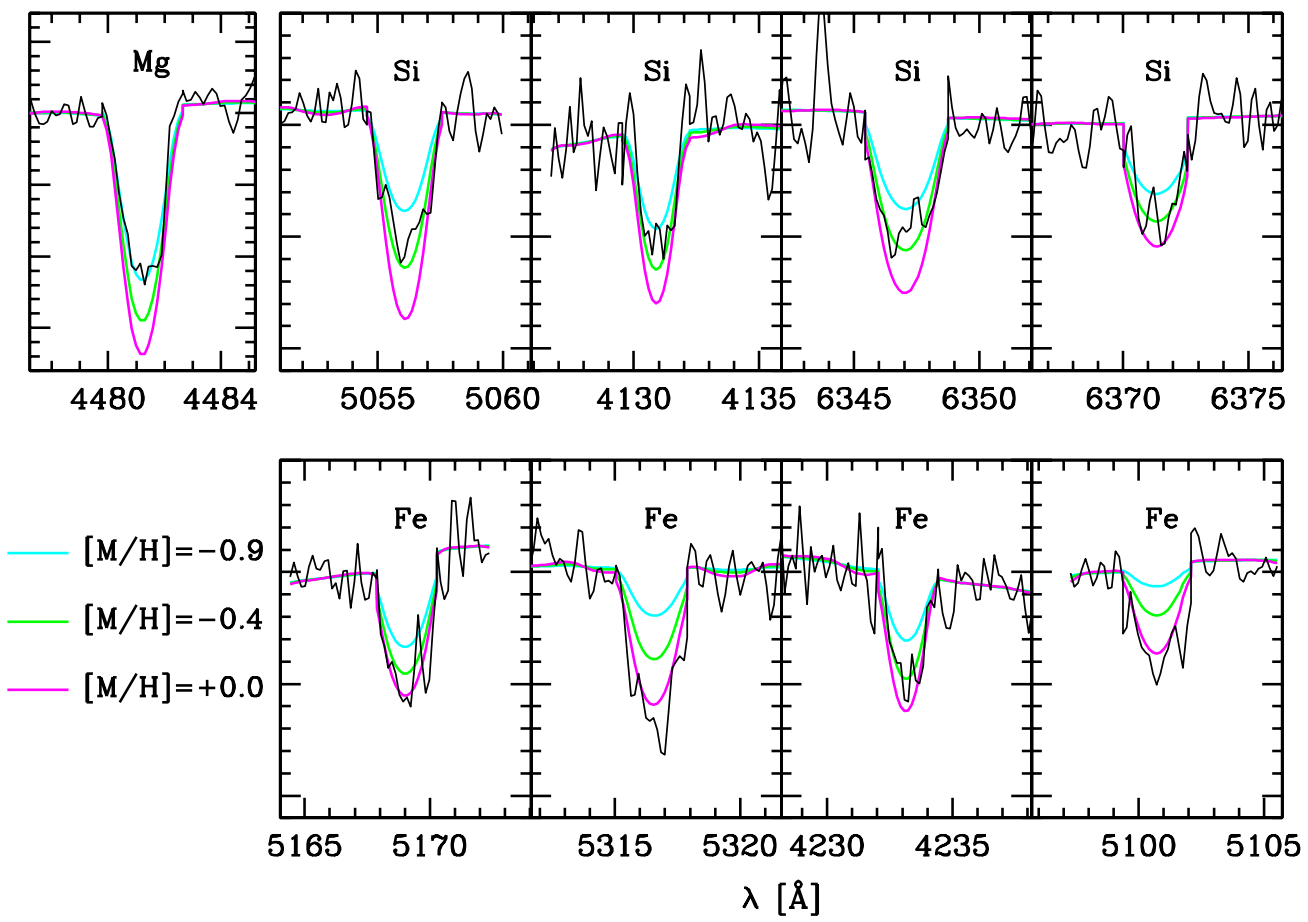

Figure 2. Strongest metal lines in the HVS17 spectrum (black lines) compared to models with $[\mathrm{M} / \mathrm{H}]=-0.9$ (cyan line), $[\mathrm{M} / \mathrm{H}]=-0.4$ (green line), and $[\mathrm{M} / \mathrm{H}]=$ +0.0 (magenta line) for the best-fit $T_{\text {eff }}, \log g$, and $v \sin i$. Mg best matches $[\mathrm{M} / \mathrm{H}]=-0.9$, the Si lines on average match $[\mathrm{M} / \mathrm{H}]=-0.4$, and the Fe lines on average match $[\mathrm{M} / \mathrm{H}]=+0.0$.

(A color version of this figure is available in the online journal.)

Table 1

Stellar Parameters

\begin{tabular}{lccc}
\hline \hline Measured & \multicolumn{3}{c}{ Derived $^{\mathrm{a}}$} \\
\hline$T_{\text {eff }}(\mathrm{K})$ & $12350 \pm 290$ & Mass $\left(M_{\odot}\right)$ & $3.91 \pm 0.09$ \\
$\log g(\mathrm{cgs})$ & $3.80 \pm 0.086$ & Radius $\left(R_{\odot}\right)$ & $4.12 \pm 0.23$ \\
$v \sin i\left(\mathrm{~km} \mathrm{~s}^{-1}\right)$ & $68.7 \pm 5.4$ & Age $(\mathrm{Myr})$ & $153 \pm 9$ \\
{$[\mathrm{C} / \mathrm{H}]$} & $-0.84 \pm 0.40$ & $M_{g}(\mathrm{mag})$ & $-1.05 \pm 0.19$ \\
{$[\mathrm{Mg} / \mathrm{H}]$} & $-0.90 \pm 0.30$ & $r_{\mathrm{GC}}(\mathrm{kpc})$ & $48.5 \pm 4.6$ \\
{$[\mathrm{Si} / \mathrm{H}]$} & $-0.39 \pm 0.18$ & $t_{\mathrm{GC}}(\mathrm{Myr})$ & $90 \pm 6$ \\
{$[\mathrm{~S} / \mathrm{H}]$} & $-0.70 \pm 0.35$ & Age $-t_{\mathrm{GC}}(\mathrm{Myr})$ & $63 \pm 11$ \\
{$[\mathrm{Ti} / \mathrm{H}]$} & $-0.90 \pm 0.30$ & & \\
{$[\mathrm{Fe} / \mathrm{H}]$} & $+0.06 \pm 0.22$ & & \\
$g_{0}(\mathrm{mag})$ & $17.428 \pm 0.015$ & & \\
$v_{\text {helio }}\left(\mathrm{km} \mathrm{s}^{-1}\right)$ & $+248.0 \pm 2.2$ & \\
\hline
\end{tabular}

Note. ${ }^{a}$ Derived quantities assume solar metallicity (see the text).

element. We exclude lines blended by another species. Figure 2 plots the strongest metal lines in the spectrum compared with some fiducial models. Iron is the best-constrained element, with 48 unblended $\mathrm{Fe}$ II lines and a weighted mean of $[\mathrm{Fe} / \mathrm{H}]=$ $+0.06 \pm 0.22$. Silicon is also well-measured, with nine unblended $\mathrm{Si}$ II lines and a weighted mean of $[\mathrm{Si} / \mathrm{H}]=-0.39 \pm 0.18$. C, $\mathrm{Mg}, \mathrm{S}$, and Ti have only a few lines and abundances ranging $-0.9<[\mathrm{M} / \mathrm{H}]<-0.7$ dex. Table 1 summarizes all of the measured parameters for HVS17.

\section{DISCUSSION}

\subsection{Stellar Nature}

HVS17 has the $T_{\text {eff }}$ and $\log g$ of either a main sequence B star or a hot BHB star. Thus we turn to metallicity and rotation to determine its nature. Main sequence B stars presumably come from metal-enriched star formation regions, whereas evolved BHB stars are normally found in old metal-poor environments like the stellar halo. Curiously, HVS17 has a solar iron abundance and sub-solar alpha abundance. Anomalous abundance patterns are seen in some hot BHB stars due to their shallow surface convection zones (Michaud et al. 2008). Yet hot BHB stars are among the slowest rotators, with observed $v \sin i \leqslant 7 \mathrm{~km} \mathrm{~s}^{-1}$ (Behr 2003a, 2003b). If HVS17 is a hot BHB star, it is the fastest rotating hot BHB star by a factor of 10. An extreme BHB rotation might be explained if the star was spun-up and ejected by a binary MBH (Löckmann \& Baumgardt 2008), however there is presently no evidence for a binary black hole in the Galactic center.

The observed $v \sin i$ of HVS17 is consistent, on the other hand, with the $v \sin i$ of late B-type main sequence stars (Abt et al. 2002; Huang \& Gies 2006). Chemically peculiar A- and B-type stars are common, because of atomic diffusion processes in the radiative atmospheres of these stars (Michaud 1970). Chemically peculiar stars are also slower rotators on average than non-peculiar main sequence stars (Smith 1996). The observed $v \sin i$ of HVS17, which is about $100 \mathrm{~km} \mathrm{~s}^{-1}$ below the mean $v \sin i$ of single late B-type main sequence stars (Abt et al. 2002; Huang \& Gies 2006), matches this expectation. Slowerthan-average rotation also fits the expectation for 3-4 $M_{\odot} \mathrm{HVS}$ ejections from a single $\mathrm{MBH}$, in which tidal synchronization of the progenitor stellar binary yields $v \sin i \sim 80 \mathrm{~km} \mathrm{~s}^{-1}$ (Hansen 2007). In either case, the observations are consistent with HVS17 being a main sequence star, and a chemically peculiar B star like HVS7 (Przybilla et al. 2008c).

Figure 3 compares the measured $T_{\text {eff }}$ and $\log g$ with Padova (Girardi et al. 2002, 2004; Marigo et al. 2008) solar metallicity (solid lines) main sequence tracks. Diffusion processes have erased any constraint provided by abundances if HVS17 is chemically peculiar. In other words, we do not know HVS17's interior composition and cannot say whether it is consistent or 


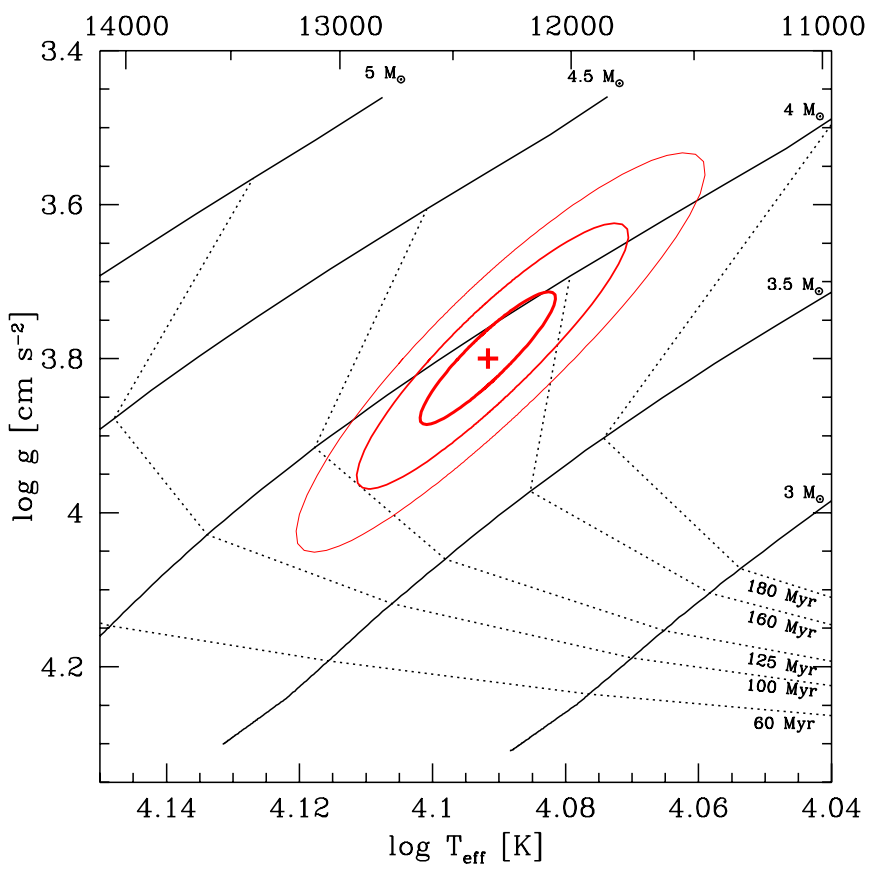

Figure 3. Measured $1 \sigma, 2 \sigma$, and $3 \sigma$ confidence regions (ellipses) compared to Marigo et al. (2008) solar metallicity main sequence tracks (straight solid lines). Isochrones (dotted lines) are plotted for the solar metallicity tracks. We conclude HVS17 is a $153 \pm 9$ Myr old $3.91 \pm 0.09 M_{\odot}$ star.

(A color version of this figure is available in the online journal.)

inconsistent with expected Galactic center abundance patterns. For discussion, we will compare with solar metallicity tracks because HVS17's iron abundance is solar. The ellipses in Figure 3 plot the measurement $1 \sigma, 2 \sigma$ and $3 \sigma$ confidence regions. Interpolating these tracks indicates that HVS17 is a $3.91 \pm 0.09 M_{\odot}$ star that is $153 \pm 9$ Myr old. The age uncertainty is relatively small because $T_{\text {eff }}$ and $\log g$ change rapidly with increasing age, as illustrated by the isochrones (dotted lines) in Figure 3. The absolute $g$-band magnitude $M_{g}=-1.05 \pm 0.19$ places HVS17 at a heliocentric distance of $49.6 \pm 4.6 \mathrm{kpc}$. Assuming the Sun is located $8 \mathrm{kpc}$ from the Galactic center, HVS17's Galactocentric distance is $r=48.5 \pm 4.6 \mathrm{kpc}$.

To estimate systematic uncertainty we compare with Bressan et al. (2012) tracks which use a different definition of solar metallicity. These tracks yield a mass of $3.71 M_{\odot}$ and an age of $175 \mathrm{Myr}$, values which differ from Marigo et al. (2008) tracks by twice our $1 \sigma$ error bars. Choice of tracks clearly introduces a systematic uncertainty: lower metallicity tracks yield lower masses, lower luminosities (and thus shorter distances and flight times), and increased ages. Fortuitously, these trends are in a direction that strengthen our conclusions below.

\subsection{Origin}

Our observations paint the following picture: HVS17 is a short-lived main sequence B star in the outer halo, traveling at an unbound radial velocity. Galactic escape velocity at $r=50 \mathrm{kpc}$ is approximately $400 \mathrm{~km} \mathrm{~s}^{-1}$ for a Milky Way halo mass of $1.6 \times 10^{12} M_{\odot}$ (Gnedin et al. 2010), and HVS17's radial velocity in the Galactic rest frame is $+445 \mathrm{~km} \mathrm{~s}^{-1}$. Two models that can explain HVS17's origin are a hyper-runaway ejection from the Galactic disk and a hypervelocity ejection from the Galactic center.

The mass and surface gravity of HVS17 yield an escape velocity from the surface of the star of $602 \mathrm{~km} \mathrm{~s}^{-1}$. If we take

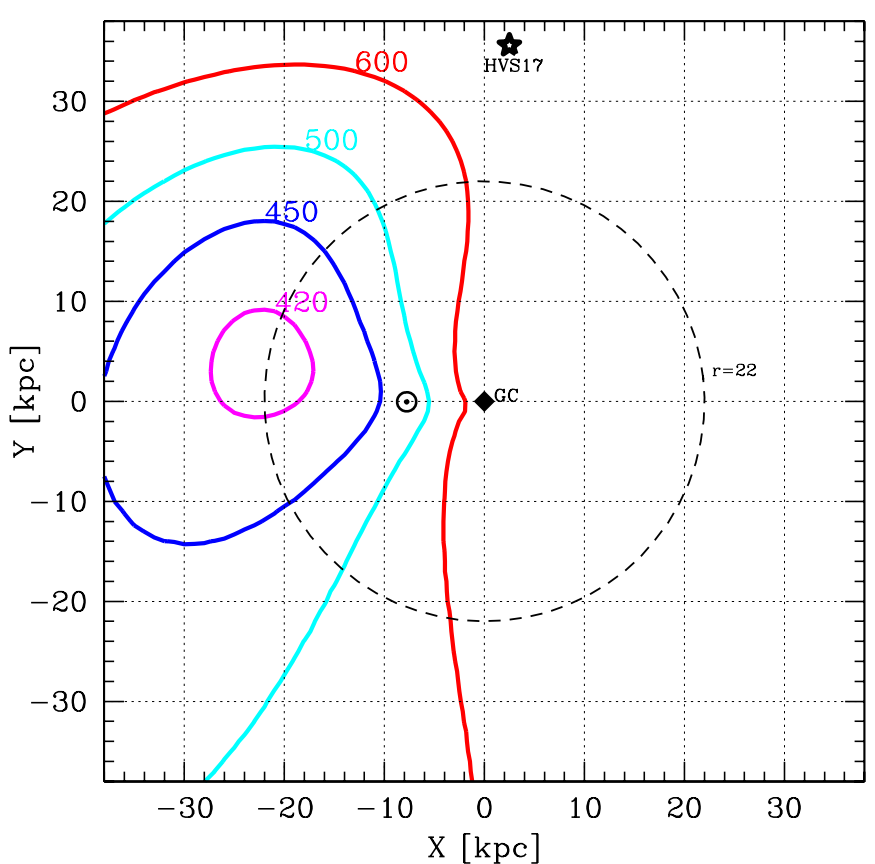

Figure 4. Galactic plane ejection velocities (contours) required to place HVS17 at its present position (star) and radial velocity. The Sun is at $\mathrm{X}=-8 \mathrm{kpc}$ and Galactic rotation is in the clockwise direction. The minimum ejection velocity is $415 \mathrm{~km} \mathrm{~s}^{-1}$ at a distance of $r=22 \mathrm{kpc}$ (dashed circle); the escape velocity from the surface of HVS17 is $600 \mathrm{~km} \mathrm{~s}^{-1}$.

(A color version of this figure is available in the online journal.)

this velocity as the speed limit on any runaway origin, whether supernovae or dynamical ejection from three- or four-body stellar encounters, then there is a finite part of the Galactic disk from which HVS17 can be ejected. We therefore use trajectory calculations to constrain HVS17's origin. Our approach is to start at the present position and radial velocity of HVS17 and calculate backward in time trajectories that cross the Galactic disk.

Figure 4 plots the ejection velocity from the Galactic disk required to place HVS17 at its present position and radial velocity. Our calculation assumes the Galactic potential model of Kenyon et al. (2008) and a $250 \mathrm{~km} \mathrm{~s}^{-1}$ circular velocity (Reid et al. 2009; McMillan \& Binney 2010). The lowest possible ejection velocity is $415 \mathrm{~km} \mathrm{~s}^{-1}$ at a distance of $r=22 \mathrm{kpc}$ from the Galactic center; ejections from inside the solar circle require velocities in excess of $500 \mathrm{~km} \mathrm{~s}^{-1}$. For context, theoretical models predict that less than $1 \%$ of runaway ejections, whether from supernovae (Portegies Zwart 2000) or dynamical ejections (Perets \& Subr 2012), have $v_{\mathrm{ej}}>200 \mathrm{~km} \mathrm{~s}^{-1}$. Extreme runaway ejection velocities require massive stars (Heber et al. 2008; Przybilla et al. 2008a; Gvaramadze 2009).

For the well-defined region in Figure 4 with $v_{\mathrm{ej}}<420 \mathrm{~km} \mathrm{~s}^{-1}$, the flight time from the disk to the location of HVS17 is $96 \pm 4$ Myr. Thus the time between when HVS17 formed and when it was ejected is $57 \pm 10 \mathrm{Myr}$ in the disk runaway scenario. This time between formation and ejection is difficult to reconcile with any runaway ejection involving massive stars that live for only 10 Myr. Ejection during the first 10 Myr of HVS17's lifetime is formally ruled out at the $5 \sigma$ level, and by a larger amount if HVS17 is a lower mass or lower metallicity (and thus older) star.

We will henceforth refer to the time between HVS17's formation and ejection as its "arrival time" (Brown et al. 2012a). For a Galactic center origin, there is no upper limit 


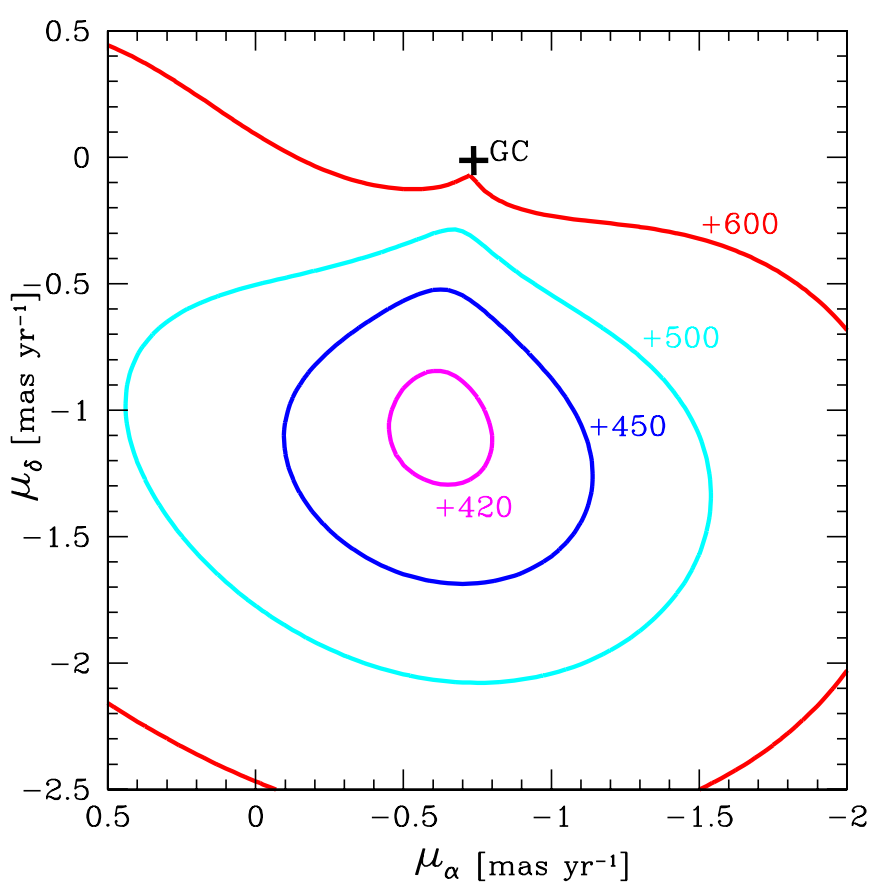

Figure 5. Predicted proper motion for a Galactic center origin (GC) or Galactic disk origins with the indicated ejection velocities (contours).

(A color version of this figure is available in the online journal.)

on arrival time. The $\mathrm{MBH}$ is always there, and on-going star formation (e.g., Lu et al. 2009) provides a constant supply of new stars. Theorists predict that dynamical interactions and orbital evolution within a triaxial potential will cause stars formed in the central region to "fill" the black hole's loss cone with timescales of 100 Myr-1 Gyr (Yu \& Tremaine 2003; Merritt \& Poon 2004; Wang \& Merritt 2004; Perets et al. 2007). Arrival time thus clearly distinguishes between the central black hole and disk runaway ejection processes.

The flight time from the Galactic center to the location of HVS17 is $90 \pm 6$ Myr. The uncertainty comes from propagating the distance and radial velocity errors through the trajectory calculation. The arrival time for the Galactic center scenario is thus $63 \pm 11$ Myr. This timescale is consistent with the timescale for stars to form in the Galactic center region and scatter into the black hole's loss cone. Three-body interactions with the MBH naturally provide unbound ejection velocities (Hills 1988; Yu $\&$ Tremaine 2003), and at a rate that is $100 \times$ larger than the ejection rate of unbound disk runaways (Brown et al. 2009; Perets \& Subr 2012).

\subsection{Proper Motion Prediction}

It appears that HVS17 comes from the Galactic center based on its unbound velocity and $\gg 10 \mathrm{Myr}$ arrival time, however, a more direct test will soon be possible: proper motion. Although

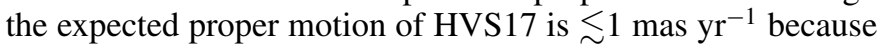
of the star's distance, the direction differs for Galactic center and Galactic disk origins.

We plot proper motions corresponding to different HVS17 trajectories in Figure 5. For a Galactic center ejection, we predict that HVS17 has $\left(\mu_{\alpha}, \mu_{\delta}\right)=(-0.75,0.0)$ mas $\mathrm{yr}^{-1}$. For a Galactic disk ejection with $v_{\mathrm{ej}}<420 \mathrm{~km} \mathrm{~s}^{-1}$, HVS17

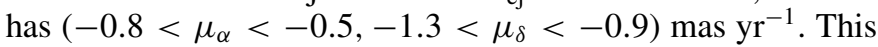
disk origin differs from the Galactic center origin by $\simeq 1$ mas $\mathrm{yr}^{-1}$. Higher disk ejection velocities allow for a broader range of proper motion, but nearly all physically possible disk ejections require trajectories with more southerly proper motions than trajectories from the Galactic center. This difference in proper motion should be easily measured by Gaia, which will achieve $\pm 0.045{\text { mas } \mathrm{yr}^{-1} \text { precision }}^{4}$ for this star.

\section{CONCLUSION}

We present Keck ESI spectroscopy of HVS17, a late B-type star traveling with a minimum Galactic rest frame velocity of $+445 \mathrm{~km} \mathrm{~s}^{-1}$. HVS17 has a projected rotation of $v \sin i=$ $68.7 \pm 5.4 \mathrm{~km} \mathrm{~s}^{-1}$ and thus is a main sequence B star. The star appears chemically peculiar with solar iron abundance and subsolar alpha abundance. Diffusion processes have thus erased any constraint provided by abundances. Comparing measured $T_{\text {eff }}$ and $\log g$ with solar metallicity stellar evolution tracks implies that HVS17 is a $3.91 \pm 0.09 M_{\odot}, 153 \pm 9$ Myr old star at a distance of $r=48.5 \pm 4.6 \mathrm{kpc}$. Sub-solar metallicity tracks systematically increase HVS17's inferred age.

We establish HVS17's origin using velocity and "arrival time," the time between its formation and subsequent ejection. A disk runaway origin suffers a fatal lifetime problem: the required $>415 \mathrm{~km} \mathrm{~s}^{-1}$ ejection velocities require massive stars that live for only $\sim 10$ Myr. For the part of the Galactic disk from which ejection velocities are less than the escape velocity from the surface of HVS17, arrival times significantly exceed $10 \mathrm{Myr}$. The central black hole origin, on the other hand, allows for any arrival time. The central black hole is also expected to eject unbound 3-4 $M_{\odot}$ stars at a rate $100 \times$ larger than disk runaway scenarios (Brown et al. 2009; Perets \& Subr 2012). We conclude that HVS17 is likely a HVS ejected by the MBH in the Galactic center.

Future proper motion measurements will directly answer the question of origin. We predict that trajectories from the

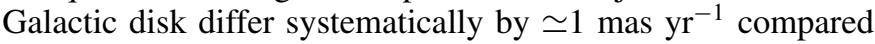
to the trajectory from the Galactic center. This difference is easily measurable with Gaia. If HVS17 is indeed ejected from the Galactic center, its proper motion, coupled with our measurement of nature and distance, will one day allow us to use it as a test particle for mapping the Milky Way's dark matter distribution.

This work was supported in part by the Smithsonian Institution. J. Cohen acknowledges partial support from AST-0908139. This research makes use of NASA's Astrophysics Data System Bibliographic Services. We are grateful to the many people who have worked to make the Keck Telescopes and their instruments a reality, and who operate and maintain these observatories. The authors extend special thanks to those of Hawaiian ancestry on whose sacred mountain we are privileged to be guests. Without their generous hospitality, none of the observations presented herein would have been possible.

Facility: Keck:II (ESI)

\section{REFERENCES}

Abt, H. A., Levato, H., \& Grosso, M. 2002, ApJ, 573, 359

Alexander, T., \& Livio, M. 2004, ApJL, 606, L21

Arzoumanian, Z., Chernoff, D. F., \& Cordes, J. M. 2002, ApJ, 568, 289

Behr, B. B. 2003a, ApJS, 149, 67

Behr, B. B. 2003b, ApJS, 149, 101

Blaauw, A. 1961, BAN, 15, 265

\footnotetext{
4 http://www.rssd.esa.int/index.php?page=Science_Performance\&project $=$ GAIA
} 
Bressan, A., Marigo, P., Girardi, L., et al. 2012, MNRAS, 427, 127

Bromley, B. C., Brown, W. R., Geller, M. J., \& Kenyon, S. J. 2009, ApJ, 706, 925

Bromley, B. C., Kenyon, S. J., Geller, M. J., \& Brown, W. R. 2012, ApJL, 749, L42

Brown, W. R., Anderson, J., Gnedin, O. Y., et al. 2010, ApJL, 719, L23

Brown, W. R., Cohen, J. G., Geller, M. J., \& Kenyon, S. J. 2012a, ApJL, 754, L2

Brown, W. R., Geller, M. J., \& Kenyon, S. J. 2009, ApJ, 690, 1639

Brown, W. R., Geller, M. J., \& Kenyon, S. J. 2012b, ApJ, 751, 55

Brown, W. R., Geller, M. J., Kenyon, S. J., \& Kurtz, M. J. 2005, ApJL, 622, L33

Brown, W. R., Geller, M. J., Kenyon, S. J., \& Kurtz, M. J. 2006a, ApJL, 640, L35

Brown, W. R., Geller, M. J., Kenyon, S. J., \& Kurtz, M. J. 2006b, ApJ, 647, 303

Brown, W. R., Geller, M. J., Kenyon, S. J., Kurtz, M. J., \& Bromley, B. C. 2007a, ApJ, 660, 311

Brown, W. R., Geller, M. J., Kenyon, S. J., Kurtz, M. J., \& Bromley, B. C. 2007b, ApJ, 671, 1708

Castelli, F., Gratton, R. G., \& Kurucz, R. L. 1997, A\&A, 318, 841

Castelli, F., \& Kurucz, R. L. 2004, arXiv:astro-ph/0405087

Dorman, B., Rood, R. T., \& O'Connell, R. W. 1993, ApJ, 419, 596

Dotter, A., Chaboyer, B., Jevremović, D., et al. 2007, AJ, 134, 376

Edelmann, H., Napiwotzki, R., Heber, U., Christlieb, N., \& Reimers, D. 2005, ApJL, 634, L181

Geier, S., Marsh, T. R., Wang, B., et al. 2013, A\&A, 554, A54

Ghez, A. M., Salim, S., Weinberg, N. N., et al. 2008, ApJ, 689, 1044

Gillessen, S., Eisenhauer, F., Trippe, S., et al. 2009, ApJ, 692, 1075

Ginsburg, I., \& Loeb, A. 2006, MNRAS, 368, 221

Girardi, L., Bertelli, G., Bressan, A., et al. 2002, A\&A, 391, 195

Girardi, L., Grebel, E. K., Odenkirchen, M., \& Chiosi, C. 2004, A\&A, 422, 205

Gnedin, O. Y., Brown, W. R., Geller, M. J., \& Kenyon, S. J. 2010, ApJL, 720, L108

Gnedin, O. Y., Gould, A., Miralda-Escudé, J., \& Zentner, A. R. 2005, ApJ, 634,344

Gray, R. O., \& Corbally, C. J. 1994, AJ, 107, 742

Gvaramadze, V. V. 2009, MNRAS, 395, L85

Hansen, B. M. S. 2007, ApJL, 671, L133

Heber, U., Edelmann, H., Napiwotzki, R., Altmann, M., \& Scholz, R.-D. 2008, A\&A, 483, L21

Hills, J. G. 1988, Natur, 331, 687

Hirsch, H. A., Heber, U., O’Toole, S. J., \& Bresolin, F. 2005, A\&A, 444, L61
Huang, W., \& Gies, D. R. 2006, ApJ, 648, 580

Humason, M. L., \& Zwicky, F. 1947, ApJ, 105, 85

Irrgang, A., Przybilla, N., Heber, U., Fernanda Nieva, M., \& Schuh, S. 2010, ApJ, 711,138

Justham, S., Wolf, C., Podsiadlowski, P., \& Han, Z. 2009, A\&A, 493, 1081

Kenyon, S. J., Bromley, B. C., Geller, M. J., \& Brown, W. R. 2008, ApJ, 680,312

Kilic, M., Gianninas, A., Brown, W. R., et al. 2013, MNRAS

Löckmann, U., \& Baumgardt, H. 2008, MNRAS, 384, 323

López-Morales, M., \& Bonanos, A. Z. 2008, ApJL, 685, L47

Lu, J. R., Ghez, A. M., Hornstein, S. D., et al. 2009, ApJ, 690, 1463

Madigan, A.-M., Levin, Y., Pfuhl, O., Genzel, R., \& Perets, H. B. 2013, ApJ, submitted

Marigo, P., Girardi, L., Bressan, A., et al. 2008, A\&A, 482, 883

McMillan, P. J., \& Binney, J. J. 2010, MNRAS, 402, 934

Merritt, D., \& Poon, M. Y. 2004, ApJ, 606, 788

Michaud, G. 1970, ApJ, 160, 641

Michaud, G., Richer, J., \& Richard, O. 2008, ApJ, 675, 1223

Perets, H. B. 2009, ApJ, 690, 795

Perets, H. B., Hopman, C., \& Alexander, T. 2007, ApJ, 656, 709

Perets, H. B., \& Subr, L. 2012, ApJ, 751, 133

Portegies Zwart, S. F. 2000, ApJ, 544, 437

Poveda, A., Ruiz, J., \& Allen, C. 1967, BOTT, 4, 860

Przybilla, N., Nieva, M. F., Heber, U., \& Butler, K. 2008a, ApJL, 684, L103

Przybilla, N., Nieva, M. F., Heber, U., et al. 2008b, A\&A, 480, L37

Przybilla, N., Nieva, M. F., Tillich, A., et al. 2008c, A\&A, 488, L51

Reid, M. J., Menten, K. M., Zheng, X. W., et al. 2009, ApJ, 700, 137

Sheinis, A. I., Bolte, M., Epps, H. W., et al. 2002, PASP, 114, 851

Smith, K. C. 1996, Ap\&SS, 237, 77

Tillich, A., Heber, U., Geier, S., et al. 2011, A\&A, 527, A137

Tillich, A., Przybilla, N., Scholz, R., \& Heber, U. 2009, A\&A, 507, L37

van Velzen, S., Farrar, G. R., Gezari, S., et al. 2011, ApJ, 741, 73

Wang, B., \& Han, Z. 2009, A\&A, 508, L27

Wang, J., \& Merritt, D. 2004, ApJ, 600, 149

Wu, X., Famaey, B., Gentile, G., Perets, H., \& Zhao, H. 2008, MNRAS, 386, 2199

Yu, Q., \& Madau, P. 2007, MNRAS, 379, 1293

Yu, Q., \& Tremaine, S. 2003, ApJ, 599, 1129

Zhang, F., Lu, Y., \& Yu, Q. 2010, ApJ, 722, 1744

Zhang, F., Lu, Y., \& Yu, Q. 2013, ApJ, 768, 153 